\title{
Crosscultural adaptation and reliability testing of the Implantable Cardioverter-Defibrillator Concerns questionnaire to optimize the care of Polish patients with implantable cardioverter-defibrillators
}

\author{
Agnieszka Młynarska1,2, Rafał Młynarski²,3, Izabella Uchmanowicz'4, Ewa Marcisz-Dyla5, Czesław Marcisz'1, Krzysztof Golba2,3 \\ 1 Department of Gerontology and Geriatric Nursing, School of Health Sciences, Medical University of Silesia, Katowice, Poland \\ 2 Department of Electrocardiology, Upper Silesian Medical Centre, Katowice, Poland \\ 3 Department of Electrocardiology and Heart Failure, School of Health Sciences, Medical University of Silesia, Katowice, Poland \\ 4 Department of Clinical Nursing, Faculty of Health Sciences, Wroclaw Medical University, Wrocław, Poland \\ 5 Department of Anxiety Disorders, Hospital of the Ministry of Internal Affairs and Administration, Katowice, Poland
}

\section{KEY WORDS}

implantable

cardioverter-

-defibrillator, internal

consistency, patient

concerns

questionnaire
Correspondence to:

Agnieszka Młynarska, PhD,

Department of Electrocardiology,

Upper Silesian Medical Center,

ul. Ziołowa 45/47,

40-635 Katowice, Poland

phone: +48322024025, email:

mlynarska83@gmail.com

Received: May 22, 2020.

Revision accepted: July 9, 2020.

Published online: July 17, 2020.

Kardiol Pol. 2020; 78 (9): 906-912

doi:10.33963/KP.15520

Copyright by the Author(s), 2020

\section{ABSTRACT}

BACKGROUND Implantable cardioverter-defibrillator (ICD) placement has now become a standard procedure in patients with ventricular arrhythmias. The primary task of an ICD is to recognize and immediately interrupt life-threatening ventricular arrhythmia via an intracardiac electrical discharge, known as a shock. AIMS The aim of this study was to adapt and test the reliability of the Polish version of the Implantable Cardioverter-Defibrillator Concerns (ICDC) questionnaire, an instrument used to determine the concerns of patients with ICDs.

METHODS Standard guidelines were applied with regard to the translation and cultural adaptation of the English version of the ICDC questionnaire in Poland. The study included 129 Polish patients with ICDs, including 28 women, at a mean (SD) age of 66.24 (12.94) years. The Cronbach a was calculated to analyze the internal consistency of the ICDC questionnaire.

RESULTS The mean ICDC score for overall concerns was 36.63 (18.56); 9.19 (5.93) for factor 1 assessing the perceived limitations, and 9.72 (5.61) for factor 2 assessing device-specific concerns. The Cronbach a reliability coefficients ranged from 0.9619 to 0.9647 , after each questionnaire item was deleted. The Cronbach a that characterized the internal consistency of the entire questionnaire was 0.96 .

CONCLUSIONS The Polish adaptation of the ICDC questionnaire proved to be a useful and quick tool to assess patients' concerns after ICD placement. Its use is recommended in the evaluation of all patients after device implantation in order to optimize therapy.

INTRODUCTION The automatic implantable cardioverter-defibrillator (ICD) was implanted for the first time on February 4, 1980 at Johns Hopkins Hospital in Baltimore, Maryland, United States. One of the pioneers of ICD therapy was Mieczysław Mirowski, born in Warsaw, Poland, in 1924. ${ }^{1,2}$ The number of patients with ICDs increases each year. Ventricular arrhythmias, the main cause of implantation, are frequent disorders and their incidence increases with age and the degree of cardiac damage. According to epidemiological data, sudden cardiac death occurs in 0.36 to 1.26 cases per 1000 people per year in the world, with a mean frequency of about 1 case per 1000 people per year. It can be assumed that sudden cardiac death affects at least 36000 to 40000 people 


\section{WHAT'S NEW?}

In this study, we present the crosscultural adaptation and test the reliability of the Implantable Cardioverter-Defibrillator Concerns questionnaire to optimize the care of Polish patients with implantable cardioverter-defibrillators.

in Poland annually, with approximately 25000 to 28000 being expected to suffer from sudden cardiac arrest due to ventricular fibrillation. Implantable cardioverter-defibrillator placement has now become a standard procedure in patients with ventricular arrhythmias. ${ }^{3,4}$ The basic and most important task of an ICD is to recognize and immediately interrupt life-threatening ventricular arrhythmia with an intracardiac electrical discharge, known as a shock. Modern cardioverter-defibrillators, apart from defibrillation and intracardiac cardioversion, have a number of additional functions, which have significantly improved patients' ability to tolerate the devices by limiting the number of high-energy discharges. Still, the experience of unpleasant shocks remains one of the basic problems for this patient population. Irritation, anxiety, bewilderment, and panic are common reactions to a defibrillator discharge, especially to the so-called electrical storm, which consists of a minimum of 3 ICD shocks per day. It is estimated that between $50 \%$ and $70 \%$ of patients sense ICD discharges within the first 2 years after implantation, and between $10 \%$ to $30 \%$ experience electrical storms. ${ }^{5,6}$ Despite the effectiveness of these devices, patients can suffer from serious psychological difficulties, mainly anxiety and depression, which contribute to the deterioration of their quality of life (QoL) and reduce the potential benefits of the therapy. ${ }^{7,8} \mathrm{Kohn}$ et al ${ }^{9}$ reported that offering psychological support prior to ICD placement, before discharge from the hospital, and during routine follow-up visits significantly reduced the levels of anxiety and depression in this population. Therefore, it is of importance to assess the severity of patients' concerns about the implanted device in the hospital and after discharge, during follow-up visits. ${ }^{9}$ The Implantable Cardioverter-Defibrillator Concerns (ICDC) questionnaire is a standardized instrument, which can be used to assess patients' concerns related to an ICD and to identify patients with ICDs who are at risk of adverse clinical outcomes. The aim of this study was to adapt and test the reliability of the Polish version of the ICDC questionnaire.

METHODS The study included 129 patients with an implanted single- or dual-chamber cardioverter-defibrillator who had been hospitalized in the Department of Electrocardiology and Heart Failure, Katowice, Poland, to exchange their ICDs.

Study participants We included 129 consecutive patients (28 women; mean [SD] age, 66.24 [12.94] years). The minimum number of participants included in the study was estimated based on the literature, which shows that the minimum sample size should be at least 5 -fold greater than the number of variables analyzed. ${ }^{4}$ In this case, the number of variables was 20 , so the minimum sample size should be 100 .

The following inclusion criteria were defined: undergoing an ICD exchange procedure due to an elective replacement indicator ${ }^{10}$ and providing consent to participate in the study. The exclusion criteria were as follows: cardiac resynchronization therapy with an ICD, diagnosed cancer in the active phase, previously diagnosed mental illness or stroke, and an incomplete questionnaire.

Participation in the study was anonymous and voluntary. The study was approved by a bioethical commission (KNW/0022/KB/36/18) and conducted in accordance with the Declaration of Helsinki and the principles of Good Clinical Practice.

Instruments Concerns about an ICD in the ICDC questionnaire are estimated using the Likert scale ranging between 0 (not at all) and 4 (very much so). This gives a possible overall score of 0 to 20 (for the number of concerns) and 0 to 80 (for increasing concerns). A higher result indicates a greater number of more serious concerns. The number of fears and increasing concerns can be used alone or in combination in order to achieve the overall number of concerns (maximum, 100). The ICDC questionnaire is divided into 2 subscales. The first subscale, factor 1 , assesses perceived limitations (questions no. 4, 5, 6, 10,11, 14, 18, 19, and 20), and the second subscale, factor 2 , device-specific concerns (questions no. 1, 6, 9, 12, 13, 15, 16, and 17). The psychometric properties of the original ICDC questionnaire are good (Cronbach $\alpha$ of 0.94 for the entire questionnaire). ${ }^{11}$

Translation process A standard 2-way procedure was used to translate (from English into Polish and then from Polish into English) and culturally adapt the ICDC into Polish. After obtaining the consent of the author of the original instrument, the questionnaire was independently translated into Polish by 2 translators. A panel of specialists verified the phrasing and meaning of all questions, as well as the clarity and correctness of the instructions. The panel consisted of 2 cardiologists, 2 cardiovascular nurses, and a specialist in the field of health psychology. Each team member had experience in working with patients with ICDs. After specialists' evaluation, the questionnaire underwent the translation process once again. Then, the initial adaptation was used in a pilot study of 30 randomly selected patients. Eventually, the final Polish version of the ICDC was obtained and analyzed. 
Statistical analysis Statistical analysis was conducted using the Polish version of the Statistica software (StatSoft, Tulsa, Oklahoma, United States). The internal consistency of the items included in the Polish version of the ICDC questionnaire was evaluated by calculating the Cronbach $\alpha$ coefficient. As recommended by Aron et al, ${ }^{12}$ the values of Cronbach $\alpha$ were regarded as optimal at $\geq 90$, good at $\geq 0.8$, acceptable at $\geq 0.7$, questionable at $\geq 0.6$, poor at $\geq 0.5$, and unacceptable at $\geq 0.5$. The ceiling and floor effects were checked to detect outliers: more than $15 \%$ of the respondents obtained the lowest or highest scores. To evaluate the goodness of fit between the models and data, we used the $\mathrm{X}^{2}$ goodness of fit, weighted root mean square residual of less than 1 , root mean square error of approximation
(RMSEA) of 0.05 or less, close fit for RMSEA, and the Tucker-Lewis index of 0.95 or greater.

RESULTS The mean (SD) ICDC score was 36.63 (18.56) (skewness, 0.2376; kurtosis, 0.3768) for overall concerns; 9.19 (5.93) for factor 1, ie, assessing the perceived limitations (skewness, 0.3786; kurtosis, 0.3932), and 9.72 (5.61) for factor 2, ie, device-specific concerns (skewness, 0.1941; kurtosis, 0.3893). The detailed characteristics of the total ICDC score are presented in TABLES 1 and 2.

Reliability The Cronbach $\alpha$ reliability coefficients ranged from 0.9619 to 0.9647 after deleting each questionnaire item. The Cronbach $\alpha$ that

TABLE 1 Statistical characteristics of the total Implantable Cardioverter-Defibrillator Concerns score and the scores of the individual factors and items

\begin{tabular}{|c|c|c|c|c|}
\hline Item & Concern ("I am worried about...") & Mean (SD) & Median (IQR) & Range \\
\hline 1 & My ICD firing & $1.17(0.78)$ & $1(1-2)$ & $0-4$ \\
\hline 2 & My ICD not working when I need it to & $1.08(0.84)$ & $1(1-1)$ & $0-4$ \\
\hline 3 & What I should do if my ICD fires & $1.12(0.82)$ & $1(1-2)$ & $0-4$ \\
\hline 4 & Doing exercise in case it causes my ICD to fire & $1.13(0.84)$ & $1(0-2)$ & $0-4$ \\
\hline 5 & Doing activities/hobbies that may cause my ICD to fire & $1.15(0.86)$ & $1(1-2)$ & $0-4$ \\
\hline 6 & My heart condition getting worse if the ICD fires & $1.24(0.88)$ & $1(1-2)$ & $0-4$ \\
\hline 7 & $\begin{array}{l}\text { The amount of time I spend thinking about my heart } \\
\text { condition and having an ICD }\end{array}$ & $1.1(0.78)$ & $1(1-2)$ & $0-4$ \\
\hline 8 & The amount of time I spend thinking about my ICD firing & $1.12(0.88)$ & $1(0-2)$ & $0-4$ \\
\hline 9 & The ICD battery running out & $1.23(0.91)$ & $1(1-2)$ & $0-4$ \\
\hline 10 & Working too hard / overdoing things causing my ICD to fire & $1.15(0.84)$ & $1(1-2)$ & $0-4$ \\
\hline 11 & Making love in case my ICD fires & $1.08(0.89)$ & $1(0-2)$ & $0-4$ \\
\hline 12 & Having no warning my ICD will fire & $1.3(0.86)$ & $1(1-2)$ & $0-4$ \\
\hline 13 & The symptoms / pain associated with my ICD firing & $1.22(0.83)$ & $1(1-2)$ & $0-4$ \\
\hline 14 & Being a burden on my partner / family & $0.86(0.89)$ & $1(0-1)$ & $0-4$ \\
\hline 15 & Not being able to prevent my ICD from firing & $1.17(0.93)$ & $1(0-2)$ & $0-4$ \\
\hline 16 & The future now that I have an ICD & $1.22(0.79)$ & $1(1-2)$ & $0-4$ \\
\hline 17 & Problems occurring with my ICD, eg, battery failure & $1.16(0.85)$ & $1(1-2)$ & $0-4$ \\
\hline 18 & Getting too stressed in case my ICD fires & $1.05(0.79)$ & $1(1-1)$ & $0-4$ \\
\hline 19 & $\begin{array}{l}\text { Not being able to work/ take part in activities and hobbies } \\
\text { because I have an ICD }\end{array}$ & $0.88(0.89)$ & $1(0-2)$ & $0-4$ \\
\hline 20 & Exercising too hard and causing my ICD to fire & $0.66(0.76)$ & $0(0-1)$ & $0-4$ \\
\hline \multicolumn{5}{|c|}{ Overall patients' scores } \\
\hline \multicolumn{2}{|c|}{ Concerns, n } & $14.54(6.17)$ & $17(12-19)$ & $0-20$ \\
\hline \multicolumn{2}{|c|}{ Increasing concerns } & $22.09(13.13)$ & $21(13-31)$ & $0-80$ \\
\hline \multicolumn{2}{|c|}{ Overall concerns } & $36.63(18.56)$ & $37(26-50)$ & $0-100$ \\
\hline \multicolumn{2}{|c|}{ Factor 1} & $9.19(5.93)$ & $8(5-14)$ & $0-36$ \\
\hline \multicolumn{2}{|c|}{ Factor 2} & $9.72(5.61)$ & $9(6-13)$ & $0-32$ \\
\hline
\end{tabular}


TABLE 2 Percentage distribution of possible answers among the components of the concerns according to the Implantable Cardioverter-Defibrillator Concerns questionnaire

\begin{tabular}{|c|c|c|c|c|c|c|}
\hline \multirow[t]{2}{*}{ Item } & \multirow[t]{2}{*}{ Concern ("I am worried about...") } & \multicolumn{5}{|c|}{ Answer } \\
\hline & & Not at all & A little & Somewhat & Quite a lot & Very much so \\
\hline 1 & My ICD firing & $24(18.6)$ & $65(50.4)$ & $34(26.3)$ & $6(4.65)$ & 0 \\
\hline 2 & My ICD not working when I need it to & $30(23.2)$ & $68(52.7)$ & $21(16.3)$ & $10(7.7)$ & 0 \\
\hline 3 & What I should do if my ICD fires & $32(24.8)$ & $55(42.6)$ & $37(28.7)$ & $5(3.7)$ & 0 \\
\hline 4 & Doing exercise in case it causes my ICD to fire & $34(26.3)$ & $48(37.2)$ & $43(33.3)$ & $4(3.1)$ & 0 \\
\hline 5 & $\begin{array}{l}\text { Doing activities / hobbies that may cause my ICD to } \\
\text { fire }\end{array}$ & $31(24)$ & $56(43.4)$ & $34(26.3)$ & $8(6.2)$ & 0 \\
\hline 6 & My heart condition getting worse if the ICD fires & $29(22.5)$ & $49(37.9)$ & $2(1.6)$ & $9(6.9)$ & 0 \\
\hline 7 & $\begin{array}{l}\text { The amount of time I spend thinking about my heart } \\
\text { condition and having an ICD }\end{array}$ & $30(23.3)$ & $56(43.4)$ & $40(31)$ & $2(1.6)$ & 0 \\
\hline 8 & $\begin{array}{l}\text { The amount of time I spend thinking about my ICD } \\
\text { firing }\end{array}$ & $36(27.9)$ & $49(37.9)$ & $37(28.7)$ & $7(5.4)$ & 0 \\
\hline 9 & The ICD battery running out & $28(21.7)$ & $57(44.2)$ & $30(23.3)$ & $14(10.8)$ & 0 \\
\hline 10 & $\begin{array}{l}\text { Working too hard / overdoing things causing my ICD } \\
\text { to fire }\end{array}$ & $31(24)$ & $54(41.9)$ & $38(29.5)$ & $6(4.6)$ & 0 \\
\hline 11 & Making love in case my ICD fires & $37(28.7)$ & $54(41.9)$ & $29(22.5)$ & $9(6.9)$ & 0 \\
\hline 12 & Having no warning my ICD will fire & $25(19.4)$ & $49(37.9)$ & $46(35.7)$ & $9(6.9)$ & 0 \\
\hline 13 & The symptoms / pain associated with my ICD firing & $26(20.2)$ & $56(43.4)$ & $40(31)$ & $7(5.4)$ & 0 \\
\hline 14 & Being a burden on my partner / family & $55(42.6)$ & $44(34.1)$ & $23(17.8)$ & $7(5.4)$ & 0 \\
\hline 15 & Not being able to prevent my ICD from firing & $33(25.6)$ & $53(41.1)$ & $32(24.8)$ & $10(7.7)$ & $1(0.77)$ \\
\hline 16 & The future now that I have an ICD & $24(18.6)$ & $57(44.2)$ & $43(33.3)$ & $5(3.9)$ & 0 \\
\hline 17 & Problems occurring with my ICD, eg, battery failure & $30(23.3)$ & $56(43.4)$ & $35(27.1)$ & $8(6.2)$ & 0 \\
\hline 18 & Getting too stressed in case my ICD fires & $31(24)$ & $67(51.9)$ & $25(19.4)$ & $6(4.6)$ & 0 \\
\hline 19 & $\begin{array}{l}\text { Not being able to work / take part in activities and } \\
\text { hobbies because I have an ICD }\end{array}$ & $54(41.9)$ & $41(31.8)$ & $29(22.5)$ & $5(3.9)$ & 0 \\
\hline 20 & Exercising too hard and causing my ICD to fire & $65(50.4)$ & $45(34.9)$ & $17(13.2)$ & $2(1.5)$ & 0 \\
\hline
\end{tabular}

Data are presented as number (percentage).

Abbreviations: see TABLE 1

characterized the internal consistency of the entire questionnaire was 0.96 . The coefficients for the total and item-total correlation, as well as those determined after deleting each item, are shown in TABLE3. For factor 1, the Cronbach $\alpha$ reliability coefficients ranged from 0.89 to 0.91 after deleting each item. The Cronbach $\alpha$ that characterized the internal consistency of factor 1 was 0.91 . In the analysis of factor 2 , the Cronbach $\alpha$ reliability coefficients ranged from 0.92 to 0.92 after deleting each item. The Cronbach $\alpha$ that characterized the internal consistency of factor 2 was 0.93 .

Confirmatory factor analysis To test the model, confirmatory factor analysis was conducted on the 20-item scale. All goodness-of-fit tests including $\mathrm{X}^{2}$ goodness of fit, weighted root mean square residual, RMSEA, close fit for RMSEA, and the Tucker-Lewis index were satisfactory (TABLE 4).
Feasibility The ceiling effect was not observed in this study, ie, none of the participants obtained the highest score (100 points), but $7.7 \%$ of the participants obtained the lowest score (0 points) representing the floor effect.

The final version of the ICDC questionnaire translated into Polish is presented in Supplementary material.

DISCUSSION The 20-item ICDC questionnaire by Frizelle ${ }^{11}$ is a tool for assessing the number and severity of patient concerns about an implanted ICD by obtaining information about life concerns. The questionnaire is recognized as a reliable scale and it can be used by healthcare workers to provide comprehensive care, identify specific problems, and perform purposeful interventions. ${ }^{13}$ Until now, there has been no psychometric tool in Poland to specifically assess 
TABLE 3 Psychometric characteristics of the Polish adaptation of the Implantable Cardioverter-Defibrillator Concerns questionnaire

\section{Item} Concern ("I am worried about...")

Corrected item-total correlation Cronbach a if item deleted

\begin{tabular}{|c|c|c|c|}
\hline 1 & My ICD firing & 0.74 & 0.96 \\
\hline 2 & My ICD not working when I need it to & 0.73 & 0.96 \\
\hline 3 & What I should do if my ICD fires & 0.82 & 0.96 \\
\hline 4 & Doing exercise in case it causes my ICD to fire & 0.81 & 0.96 \\
\hline 5 & Doing activities / hobbies that may cause my ICD to fire & 0.76 & 0.96 \\
\hline 6 & My heart condition getting worse if the ICD fires & 0.81 & 0.96 \\
\hline 7 & $\begin{array}{l}\text { The amount of time I spend thinking about my heart condition } \\
\text { and having an ICD }\end{array}$ & 0.72 & 0.96 \\
\hline 8 & The amount of time I spend thinking about my ICD firing & 0.76 & 0.96 \\
\hline 9 & The ICD battery running out & 0.75 & 0.96 \\
\hline 10 & Working too hard/overdoing things causing my ICD to fire & 0.83 & 0.96 \\
\hline 11 & Making love in case my ICD fires & 0.75 & 0.96 \\
\hline 12 & Having no warning my ICD will fire & 0.78 & 0.96 \\
\hline 13 & The symptoms / pain associated with my ICD firing & 0.8 & 0.96 \\
\hline 14 & Being a burden on my partner / family & 0.61 & 0.96 \\
\hline 15 & Not being able to prevent my ICD from firing & 0.8 & 0.96 \\
\hline 16 & The future now that I have an ICD & 0.73 & 0.96 \\
\hline 17 & Problems occurring with my ICD, eg, battery failure & 0.73 & 0.96 \\
\hline 18 & Getting too stressed in case my ICD fires & 0.76 & 0.96 \\
\hline 19 & $\begin{array}{l}\text { Not being able to work / take part in activities and hobbies } \\
\text { because I have an ICD }\end{array}$ & 0.64 & 0.96 \\
\hline 20 & Exercising too hard and causing my ICD to fire & 0.58 & 0.96 \\
\hline
\end{tabular}

Abbreviations: see TABLE1

TABLE 4 Goodness-of-fit indices of the model for the Implantable Cardioverter-Defibrillator Concerns questionnaire

\begin{tabular}{|c|c|c|c|c|c|c|c|}
\hline \multirow[t]{2}{*}{ Model } & \multicolumn{3}{|c|}{$\chi^{2}$ goodness of fit } & \multicolumn{3}{|c|}{ RMSEA } & \multirow[t]{2}{*}{ TLI } \\
\hline & $\chi^{2}$ & $d f$ & $P$ value & RMSEA & $95 \% \mathrm{CI}$ & CFit & \\
\hline ICDC & 40.05 & 20 & 0.0049 & 0.0477 & $0-0.095$ & 0.405 & 0.903 \\
\hline
\end{tabular}

Abbreviations: CFit, close fit using RMSEA; $d f$, degrees of freedom; ICDC, Implantable Cardioverter-Defibrillator Concerns; RMSEA, root mean square error of approximation; TLI, Tucker-Lewis index

the level of patient functioning after ICD placement, after ICD discharges, and even after electrical storms. The significance of using questionnaires in particular patient groups is indicated by numerous publications presenting language adaptations of the scales to the Polish conditions, including the Arrhythmia-Specific Questionnaire in Tachycardia and Arrhythmia. ${ }^{14}$

The purpose of our study was to adapt the ICDC questionnaire to the conditions of the Polish population and to assess the reliability of this questionnaire in patients with ICDs. Testing the reliability of the questionnaire was based on establishing the standard psychometric features of the scale, while the Cronbach $\alpha$ coefficient was considered to be a measure of its internal consistency. The internal consistency of the Polish ICDC adaptation was 0.96, which was similar to that of the original instrument (0.94). The values of the internal consistency in validation studies of other language versions are not available in any of the worldwide references. Actually, the most frequent validated tool is a shortened version of the questionnaire-developed by Susanne Pedersen ${ }^{15,16}$ and consisting of 8 items selected from the original 20-item questionnaire.

Educating patients with ICDs is a crucial element of the therapeutic plan. The patient should receive information about the way of ICD functioning, care of the postoperative wound, guidelines for dealing with household appliances, and 
returning to work and physical activity. The analysis of patients' fears at this stage will enable healthcare professionals to adjust education to patients' needs and plan targeted education to address the problems of an individual. This will help to eliminate problems at a later stage of the ICD patient's functioning. Not dealing with the problems of patients with ICDs or limitations on their activities, eg, hobbies, physical activity, or sexual activity, is not a solution, as it only causes frustration associated with these limitations and deterioration of QoL and disease acceptance. ${ }^{17-19}$

Psychiatric anxiety is not a rare problem in patients undergoing long-term ICD therapy. The prevalence of anxiety and concerns in ICD patients is increasing, and the implantation guidelines are being expanded to include prevention of sudden cardiac death in high-risk patients. ${ }^{20}$ Although numerous studies have confirmed that ICD placement improves patients' QoL, the problems associated with implantation, frequent shocks, and a poor understanding of the therapy by a patient may exacerbate their anxiety and depression. Implantable cardioverter-defibrillator placement and therapy can also cause strong anger, stress, and fear in patients receiving treatment. ${ }^{21}$ In secondary prevention of cardiovascular diseases, patients presenting risk factors for depression are more frequently monitored and they are less often willing to change their lifestyles and comply with therapeutic recommendations and requirements. ${ }^{22}$

Numerous ICD discharges are a relevant negative factor contributing to mental disorders and lowered QoL. Decline in psychosocial functioning and QoL is a common observation after the occurrence of an ICD shock. ${ }^{23}$ Even patients who have not experienced an ICD discharge may have difficulties with adjusting to the new situation owing to fear of shock, which can cause increased anxiety, withdrawal behavior, and negative perception of self-efficacy. ${ }^{24}$

Markers of psychosocial needs in patients with ICDs include: younger age (below 50 years), insufficient knowledge of the condition of the heart and / or an ICD, a significant history of psychological problems, poor social support, and comorbidities. Previous studies have reported that the incidence of depression is generally higher in women than in men. Rahmawati et al ${ }^{26}$ demonstrated this finding in a cohort study of patients with ICDs in Japan. In that study, women showed a higher incidence of depression and were more worried about ICDs than men. ${ }^{25,26}$

Conclusions Our study showed that the tested questionnaire is valid and reproducible and can be used to assess the concerns of patients with ICDs in Poland. The Polish adaptation of the ICDC questionnaire has proven to be a useful and quick tool to assess patient concerns after
ICD placement. It is recommended to be used in the assessment of all patients after device implantation in order to optimize their therapy.

\section{SUPPLEMENTARY MATERIAL}

Supplementary material is available at www.mp.pl/kardiologiapolska.

\section{ARTICLE INFORMATION}

ACKNOWLEDGMENTS We would like to thank Dr Dorothy Frizelle for permission to use the original ICDC questionnaire. This study was funded by Medical University of Silesia (no. KNW-2-Z07/N/8/N; to AM).

\section{CONFLICT OF INTEREST None declared.}

OPEN ACCESS This is an Open Access article distributed under the terms of the Creative Commons Attribution-NonCommercial-NoDerivatives 4.0 International License (CC BY-NC-ND 4.0), allowing third parties to download articles and share them with others, provided the original work is properly cited, not changed in any way, distributed under the same license, and used for noncommercial purposes only. For commercial use, please contact the journal office at kardiologiapolska@ptkardio.pl.

HOW TO CITE Młynarska A, Młynarski R, Uchmanowicz I, et al. Crosscultural adaptation and reliability testing of the Implantable Cardioverter-Defibrillator Concerns questionnaire to optimize the care of Polish patients with implantable cardioverter-defibrillators. Kardiol Pol. 2020; 78: 906-912. doi:10.33963/ KP.15520

\section{REFERENCES}

1 Mirowski M. The automatic implantable cardioverter-defibrillator: an overview. J Am Coll Cardiol. 1985; 6: 461-466.

2 May CD, Smith PR, Murdock CJ, Davis MJ. The impact of the implantable cardioverter defibrillator on quality-of-life. Pacing Clin Electrophysiol. 1995; 18: 1411-1418.

3 Timmis A, Townsend N, Gale C, et al. ESC Scientific Document Group. European Society of Cardiology: cardiovascular disease statistics 2017. Eur Heart J. 2018; 39: 508-579.

4 Hindricks G, Camm J, Merkely B, et al. The EHRA White Book 2017. The current status of cardiac electrophysiology in ESC member countries. Tenth edition. 2017: 396-404.

5 Glikson M, Friedman P. The implantable cardioverter defibrillator. Lancet. 2001; 357: 1107-1117.

6 Sears $\mathrm{SJr}$, Conti J. Understanding implantable cardioverter defibrillator shocks and storms: medical and psychosocial considerations for research and clinical care. Clin Cardiol. 2003; 26: 107-111.

7 Bostwick J, Sola C. An updated review of implantable cardioverter/defibrillators, induced anxiety, and quality of life. Heart Fail Clin. 2011; 7: 101-108.

8 Rottmann N, Skov 0, Andersen C, et al. Psychological distress in patients with an implantable cardioverter defibrillator and their partners. J Psychosom Res. 2018; 113: 16-21.

9 Kohn C, Petrucci R, Baessler C, et al. The effect of psychological intervention on patients' long-term adjustment to the ICD: a prospective study. Pacing Clin Electrophysiol. 2000; 23: 450-456.

10 Ponikowski P, Voors A, Anker S, et al. 2016 ESC Guidelines for the diagnosis and treatment of acute and chronic heart failure: the Task Force for the diagnosis and treatment of acute and chronic heart failure of the European Society of Cardiology (ESC) developed with the special contribution of the Heart Failure Association (HFA) of the ESC. Eur Heart J. 2016; 37: 2129-2200.

11 Frizelle D, Lewin B, Kaye G, Moniz-Cook E. Development of a measure of the concerns held by people with implanted cardioverter defibrillators: the ICDC. Br J Health Psychol. 2006; 11: 293-301.

12 Aron A, Aron E, Coups E. Statistics for Psychology. 5th ed. Pearson International Edition. Pearson; 2008: 619.

13 Frizelle $D$, Lewin $R$, Kaye $G$, et al. Cognitive-behavioural rehabilitation programme for patients with an implanted cardioverter defibrillator: a pilot study. $\mathrm{Br}$ J Health Psychol. 2004; 9: 381-392.

14 Lomper K, Sławuta A, Dudek K, et al. Psychometric evaluation of the Polish version of the arrhythmia-specific questionnaire in tachycardia and arrhythmia: a new tool for symptom and health-related quality of life assessment. Kardiol Pol. 2019; 77: 541-552.

15 Pedersen S, van Domburg R, Theuns D, et al. Concerns about the implantable cardioverter defibrillator: a determinant of anxiety and depressive symptoms independent of experienced shocks. Am Heart J. 2005; 149: 664-669.

16 Pedersen S, Van Domburg R, Theuns D, et al. Type D personality is associated with increased anxiety and depressive symptoms in patients with an implantable cardioverter defibrillator and their partners. Psychosom Med. 2004; 66: 714-719.

17 Saksena S, Madan N. Management of the patient with an implantable cardioverter-defibrillator in the third millennium. Circulation. 2002; 106: 2642-2646. 
18 Sears S Jr, Conti J. Quality of life and psychological functioning of ICD patients. Heart. 2002; 87: 488-493.

19 Haugaa K, Potpara T, Boveda S, et al. Patients' knowledge and attitudes regarding living with implantable electronic devices: results of a multicenter, multinational patient survey conducted by the European Heart Rhythm Association. Europace. 2018; 20: 386-391.

20 Ding L, Hua W, Niu H, et al. Primary prevention of sudden cardiac death using implantable cardioverter defibrillators. Europace. 2008; 10: 1034-1041.

21 Baskar S, Jefferies J, Salberg L, et al. Patient understanding of disease and the use and outcome of implantable cardioverter defibrillators in hypertrophic cardiomyopathy. Pacing Clin Electrophysiol. 2018; 41: 57-64

22 Kozela M, Doryńska A, Misiowiec W, et al. Do participants with depression receive more counseling on cardiovascular disease risk factors? The results of the WOBASZ II Study. Kardiol Pol. 2019; 77: 1176-1181.

23 Craney J, Mandle C, Munro B, et al. Implantable cardioverter defibrillators: physical and psychosocial outcomes. Am. J. Crit. Care. 1997; 6: 445-451

24 Hirsh A, Sears S, Conti]. Cognitive and behavioral treatments for anxiety and depression in a patient with an implantable cardioverter defibrillator (ICD): a case report and clinical discussion. J Clin Psychol Med Settings. 2009; 16: 270-279.

25 Kessler R, Berglund P, Demler 0, et al. The epidemiology of major depressive disorder: results from the National Comorbidity Survey Replication (NCSR). JAMA. 2003; 289: 3095-3105.

26 Rahmawati A, Chishaki A, Sawatari $\mathrm{H}$, et al. Gender disparities in quality of life and psychological disturbance in patients with implantable cardioverter-defibrillators. Circ J. 2013; 77: 1158-1165. 